\title{
Phase Retrieval by Alternating Minimization with Random Initialization
}

\author{
Teng Zhang
}

\begin{abstract}
We consider a phase retrieval problem, where the goal is to reconstruct a $n$-dimensional complex vector from its phaseless scalar products with $m$ sensing vectors, independently sampled from complex normal distributions. We show that, with a random initialization, the classical algorithm of alternating minimization succeeds with high probability as $n, m \rightarrow \infty$ when $m / \log ^{3} m \geq M n^{3 / 2} \log ^{1 / 2} n$ for some $M>0$. This is a step toward proving the conjecture in [27], which conjectures that the algorithm succeeds when $m=O(n)$. The analysis depends on an approach that enables the decoupling of the dependency between the algorithmic iterates and the sensing vectors.
\end{abstract}

\section{INTRODUCTION}

$\mathbf{T}$ HIS article concerns the phase retrieval problem as follows: let $\mathbf{z} \in \mathcal{C}^{n}$ be an unknown vector, and given $m$ known sensing vectors $\left\{\mathbf{a}_{i}\right\}_{i=1}^{m} \in \mathcal{C}^{n}$, we have the observations

$$
y_{i}=\left|\mathbf{a}_{i}^{*} \mathbf{z}\right|, i=1,2, \cdots, m \text {. }
$$

Then can we reconstruct $\mathbf{z}$ from the observations $\left\{y_{i}\right\}_{i=1}^{m}$ ? In this work, we assume that the sensing vectors $\left\{\mathbf{a}_{i}\right\}_{i=1}^{m}$ are sampled from a complex normal distribution $C N(0, \mathbf{I})$. That is, both its real component and its imaginary component follows from a real Gaussian distribution of $N(0, \mathbf{I} / 2)$.

This problem is motivated from the applications in imaging science, and we refer interested readers to [23], [6] for more detailed discussions on the background in engineering and additional applications in other areas of sciences and engineering.

Because of the practical ubiquity of the phase retrieval problem, many algorithms and theoretical analysis have been developed for this problem. For example, an interesting recent approach is based on convex relaxation [8], [7], [28], that replaces the non-convex measurements by convex measurements through relaxation. Since the associated optimization problem is convex, it has properties such as convergence to the global minimizer, and it has been shown that under some assumptions on the sensing vectors, this method recovers the correct $\mathbf{z}$ [5], [16]. However, since these algorithms involve semidefinite programming for $n \times n$ positive semidefinite matrices, the computational cost could be prohibitive when $n$ is large. Recently, several works [1], [15], [17], [18], [22] proposed and analyzed an alternate convex method that uses linear programming instead of semidefinite programming, which is more computationally efficient, but the program itself requires an "anchor vector", which needs to be a good approximate estimation of z.

Another line of works are based on Wirtinger flows, i.e., gradient flow in the complex setting [6], [9], [30], [31], [4], [29], [24], [10]. Some theoretical justifications are also provided [6], [24]. However, since the objective functions are nonconvex, many of these algorithms require careful initializations, which are usually only justified when the measurement vectors follow a very specific model. In addition, there are technical issues in implementation such as choosing step sizes, which makes the implementation slightly more complicated. In particular, most theoretical analysis simply assume sufficiently small step sizes, which does give a clear guidance to practice.

The most widely used method is perhaps the alternate minimization (Gerchberg-Saxton) algorithm and its variants [14], [12], [13], that is based on alternating projections onto nonconvex sets [2]. As a result, in some literature it is also called the alternating projection method [27]. This method is very simple

T. Zhang was with the Department of Mathematics, University of Central Florida, Orlando, FL, 32765 USA e-mail: teng.zhang@ucf.edu. 
to implement and is parameter-free. However, since it is a nonconvex algorithm, its properties such as convergence are only partially known. Netrapalli et al. [21] studied a resampled version of this algorithm and established its convergence as the number of measurements $m$ goes to infinity when the measurement vectors are independent standard complex normal vectors. Marchesini et al. [19] studied and demonstrated the necessary and sufficient conditions for the local convergence of this algorithm. Recently, Waldspurger [27] showed that when $m \geq C n$ for sufficiently large $C$, the alternating minimization algorithm succeeds with high probability, provided that the algorithm is carefully initialized. This work also conjectured that the alternate minimizations algorithm with random initialization succeeds with $m \geq C n$.

One particular difficulty in the analysis of the alternating minimization algorithm is the stationary points. Currently,most papers on nonconvex algorithms depend on the analysis showing that all (attractive) stationary points of the algorithm are well-behaved in the sense that it is the desired solution, or close to the desired solution, for example, [25]. Then the standard algorithm such as gradient descent algorithm or trust-region method can be applied to the problem to obtain the stationary point. However, as pointed out in [27], in the regime $m=O(n)$, the alternating minimization algorithm has attractive stationary points that are not the desired solution. While empirically these undesired stationary points are not obstacles for the success of the algorithm since their attraction basins seem small, but it prevents us from applying the common approach of analyzing stationary points.

Recently, [32] shows that the algorithm improves the correlation between the estimator and the truth in each iteration with high probability. Based on this observation, it shows that a resampled version of the alternating minimization algorithm converges to the solution with high probability when $m=O\left(n \log ^{5} n\right)$. However, this approach can not be applied to analyze the alternating minimization algorithm directly, since the estimator at the $k$-th iteration is correlated with the sensing vectors. As a result, to analyze the nonresampled version, one needs to find a way to decouple the estimator at the $k$-th iteration and the sensing vectors.

The contribution of this work is to show that the alternating minimization algorithm with random initialization succeeds with high probability when $m / \log ^{3} m>M n^{1.5} \log ^{0.5} n$. While it still does not match the conjecture of $m=O(n)$, it is the best result on the classic algorithm with random initialization and without any resampling or construction of a good initialization yet. Compared with [32], the novelty in this analysis is the decoupling of the sensing vectors and the estimator at the $k$-th iteration. The approach fixes the first $k-1$ algorithmic iterates are fixed and analyzes the conditional distribution of the sensing vectors. This approach, inspired by the analysis of LASSO in [3], is the main technical contribution of this work. In spirit, this contribution is very similar to leave-one-out approach that also enables decoupling in [10], and based on their leave-one-out approach, they show that an algorithm for the phase retrieval converges linearly. However, the analyzed algorithm is very different and their work assumes that the sensing vectors and the $\mathbf{z}$ are real-valued. In addition, it seems more difficult to apply the leave-one-out approach here, as the iterations is a little bit more complicated.

The paper is organized as follows. Section $[-\mathrm{B}$ presents the algorithm and the main results of the paper, Theorem I.1. The proof of Theorem I.1 is given in Section II, where the main proof of Theorem I.1 is given in Section II-B, the proof of the main lemmas are given in Section II-C, and the auxiliary lemmas and their proofs are given in Section II-D

\section{A. Notations}

For any $z \in \mathcal{C},|z|$ represents the modulus of $z$. We use $\operatorname{Sp}\left(\mathbf{a}_{1}, \cdots, \mathbf{a}_{n}\right)$ to represent the subspace spanned by $\mathbf{a}_{1}, \cdots, \mathbf{a}_{n}$, i.e., the set $\left\{\mathbf{x}: \mathbf{x}+\sum_{i=1}^{n} c_{i} \mathbf{a}_{i}\right.$, for $\left.c_{1}, \cdots, c_{n} \in \mathcal{C}\right\}$. Note that here the subspace is slightly different from the standard subspace, where the coefficient of each vector is a complex number. We use $P_{L}$ to denote the projection onto the subspace $L: P_{L}(\mathbf{z})$ represents the nearest point on $L$ to $\mathbf{z}$.

For any $z \in \mathcal{C}$, phase $(z)=z /|z|$ is the phase of $z$. For any vector $\mathbf{z}=\left(z_{1}, \cdots, z_{m}\right)$, phase $(\mathbf{z})$ is the phases for each elements:

$$
\operatorname{phase}(\mathbf{z})=\left(\operatorname{phase}\left(z_{1}\right), \cdots, \operatorname{phase}\left(z_{m}\right)\right)
$$


We use $\odot$ to denote the pointwise product between the phase of the first vector and the modulus of the second vector. That is,

$$
(\mathbf{w} \odot \mathbf{y})_{i}=\frac{w_{i}}{\left|w_{i}\right|}\left|y_{i}\right|
$$

For any vector $\mathbf{z} \in \mathcal{C}^{m},\|\mathbf{z}\|$ represents its Euclidean norm: $\|\mathbf{z}\|=\sqrt{\sum_{i=1}^{m}\left|z_{i}\right|^{2}}$, and its 1-norm and $\infty$-norm are defined by $\|\mathbf{z}\|_{1}=\sum_{i=1}^{m}\left|z_{i}\right|$ and $\|\mathbf{z}\|_{\infty}=\max _{1 \leq i \leq m}\left|z_{i}\right|$.

\section{B. Algorithm and Main result}

The alternating minimization method is one of the earliest methods that was introduced for phase retrieval problems [14], [12], [13], and it is based on alternating projections onto nonconvex sets [2]. Let $\mathbf{A} \in \mathcal{C}^{m \times n}$ be a matrix with columns given by $\mathbf{a}_{1}, \mathbf{a}_{2}, \cdots, \mathbf{a}_{m}$, then its goal is to find a vector in $\mathcal{C}^{m}$ such that it lies in both the subspace $L=\operatorname{range}(\mathbf{A}) \in \mathcal{C}^{m}$ and the set of correct amplitude $\mathcal{A}=\left\{\mathbf{w} \in \mathcal{C}^{m}:\left|\mathbf{w}_{i}\right|=y_{i}\right\}$. For this purpose, the algorithm picks an initial guess $\mathbf{x}^{(1)}$ in $\mathcal{C}^{n}$ and alternatively project $\mathbf{A} \mathbf{x}^{(1)}$ on the both sets. Let $\mathbf{w}^{(k)}=\mathbf{A x}^{(k)}$ for all $k \geq 1$, then the projections $P_{L}, P_{\mathcal{A}}: \mathcal{C}^{m} \rightarrow \mathcal{C}^{m}$ can be defined by

$$
P_{L}(\mathbf{w})=\mathbf{A}\left(\mathbf{A}^{*} \mathbf{A}\right)^{-1} \mathbf{A}^{*} \mathbf{w}, \quad\left[P_{\mathcal{A}}(\mathbf{w})\right]_{i}=y_{i} \frac{\mathbf{w}_{i}}{\left|\mathbf{w}_{i}\right|},
$$

and the alternating minimization algorithm is given by applying the operator $P_{L} P_{\mathcal{A}}$ recursively to the vector $\mathbf{w}^{(1)}$, i.e.,

$$
\mathbf{w}^{(k+1)}=P_{L} P_{\mathcal{A}} \mathbf{w}^{(k)} .
$$

Then the estimator of $\mathbf{x}$ at the $k$-th iteration is obtained by solving $\mathbf{x}^{(k)}=\mathbf{A} \mathbf{w}^{(k)}$.

This algorithm has been studied in [27] and Theorem 2 in [27] shows the convergence of the algorithm if $m>M n$ and if there is a good initialization. In addition, it conjectures that random initialization also succeed in this setting. In this article, we prove that this conjecture holds when $m / \log ^{2} m>M n^{1.5} \log ^{0.5} n$ for some $M>0$. The rigorous statement is as follows:

Theorem I.1. Assuming that the sensing vectors $\left\{\mathbf{a}_{i}\right\}_{i=1}^{m}$ are i.i.d. sampled from the complex normal distribution $C N(0, \mathbf{I})$, then there exists $M>0$ such that if $m / \log ^{3} m \geq M n^{3 / 2} \log ^{1 / 2} n$, then the alternating projection algorithm with random initialization (obtained from a uniform distribution on the sphere of $\left.\mathcal{C}^{n}\right)$ succeeds almost surely in the sense that as $n, m \rightarrow \infty$,

$$
\operatorname{Pr}\left(\lim _{k \rightarrow \infty} \inf _{\psi \in \mathbb{R}}\left\|e^{i \psi} \mathbf{x}^{(k)}-\mathbf{z}\right\|=0\right) \rightarrow 1 .
$$

In the proof, for simplicity when we talk about a "random unit vector in $\mathcal{C}^{m} /$ subspace $L$ ", we implicitly assume that it is sampled from the uniform distribution on the unit sphere in $\mathcal{C}^{m}$ or subspace $L$. The constants $c, C$ are used to represent a constant that is independent of $m$ and $n$, and it is used to represent different constants in different equations. In addition, since the theorem focus on the setting when $n$ and $m$ both large, we write down inequalities under this assumption. For example, we may write $\log ^{3} n<n$ even though it only holds for large $n$.

\section{PROOF OF THEOREMI.1}

In the proof, we will first present reduced form of the statement of Theorem I.1 in Section II-A, and then present the proof of this reduced statement in Section II-B. The proof of the main lemmas are given in Section $\Pi \mathrm{II}-\mathrm{C}$, and the auxiliary lemmas (which are mostly generic results on measure concentration) and their proofs are given in Section II-D 


\section{A. An Equivalent form of Theorem I.1}

In this section we first introduce a few assumptions on $\mathbf{A}$ and some modification of the algorithm, which does not impact the performance of the algorithm but would simplify the proof later.

First, we investigate the performance of the same algorithm if the sensing matrix $\mathbf{A}$, the underlying signal $\mathbf{z}$ and the initialization $\mathbf{x}^{(1)}$ are replaced by $\tilde{\mathbf{A}}=\mathbf{A D}, \tilde{\mathbf{z}}=\mathbf{D}^{-1} \mathbf{z}$, and $\tilde{\mathbf{x}}^{(1)}=\mathbf{D}^{-1} \mathbf{x}^{(1)}$ respectively, for some $\mathbf{D} \in \mathcal{C}^{n \times n}$. Then $\mathbf{w}^{(1)}$ and $\mathbf{y}$ are unchanged, and $\operatorname{range}(\tilde{\mathbf{A}})=\operatorname{range}(\mathbf{A})$, which means that the updates in (1) is unchanged, and the estimators between these two settings have the connection of $\tilde{\mathbf{x}}^{(k)}=\mathbf{D}^{-1} \mathbf{x}^{(k)}$. As a result, $\left\|e^{i \psi} \tilde{\mathbf{x}}^{(k)}-\tilde{\mathbf{z}}\right\| \rightarrow 0$ if and only if $\left\|e^{i \psi} \mathbf{X}^{(k)}-\mathbf{z}\right\| \rightarrow 0$. For the rest of the proof, we will analyze an equivalent problem, where $\mathbf{D}=\left(\mathbf{A}^{*} \mathbf{A}\right)^{-1 / 2}$ and $\mathbf{A}$ is replaced with $\mathbf{A}\left(\mathbf{A}^{*} \mathbf{A}\right)^{-1 / 2}$, the projection matrix to the subspace $L$.

Second, WLOG we assume that $\|\mathbf{z}\|=1$ (which implies that $\|\mathbf{y}\|=1$ because $\mathbf{A}$ is a projection matrix) and we normalize $\mathbf{w}$ in the update formula (1):

$$
\mathbf{w}^{(k+1)}=\frac{P_{L} P_{\mathcal{A}} \mathbf{w}^{(k)}}{\left\|P_{L} P_{\mathcal{A}} \mathbf{w}^{(k)}\right\|} .
$$

Compared with the original form (1), $\mathbf{w}^{(k)}$ is normalized to a unit vector in each iteration. Since the operator $P_{\mathcal{A}}$ is invariant to the scaling, and $\mathbf{w}^{(k+1)}$ depends on $\mathbf{w}^{(k)}$ through $P_{\mathcal{A}} \mathbf{w}^{(k)}$, the alternating minimization algorithm with normalization (3) is equivalent to the standard version (1) with a "correct" scaling, and it is relatively straightforward to verify that Theorem I.1 holds for (3) if and only if it holds for (1).

Since $\left\{\mathbf{a}_{i}\right\}_{i=1}^{n}$ are i.i.d. sampled from $C N\left(0, \mathbf{I}_{m \times m}\right), L$ is a random $n$-dimensional subspace in $\mathcal{C}^{m}$. Combining the analysis above, to prove Theorem I.1. we will address the following equivalent problem:

- Choose a unit vector $\mathbf{z} \in \mathcal{C}^{n}$ and a random $n$-dimensional subspace $L$ in $\mathcal{C}^{m}$, and a random unit vector on $L$, denote it by $\mathbf{w}^{(1)}$. Let $\mathbf{y}=\left|\boldsymbol{\Pi}_{L}^{*} \mathbf{z}\right|$, where $\boldsymbol{\Pi}_{L} \in \mathcal{C}^{m \times n}$ represents a random projection matrix to $L$ (there are many choices of $\Pi_{L}$ : for any unitary matrix $\mathbf{U} \in \mathcal{C}^{n \times n}, \Pi_{L} \mathbf{U}$ is another projection matrix to $L$, and we randomly choose one).

- The iterative update formula is given by

$$
\mathbf{w}^{(k+1)}=\frac{P_{L}\left[\mathbf{w}^{(k)} \odot \mathbf{y}\right]}{\left\|P_{L}\left[\mathbf{w}^{(k)} \odot \mathbf{y}\right]\right\|},
$$

and $\mathbf{x}^{(k)}=\Pi_{L}^{*} \mathbf{w}^{(k)}$.

- Goal: prove (2).

\section{B. Main Proof}

In the proof, we first define a set of orthogonal unit vectors in $\mathcal{C}^{m}$ :

$$
\begin{aligned}
& \mathbf{u}_{0}=\Pi_{L}^{*} \mathbf{z}, \text { (note that }\left\|\mathbf{u}_{0}\right\|=1 \text { since }\|\mathbf{z}\|=1 \text { ) } \\
& \mathbf{u}_{k}=\frac{\mathbf{w}^{(k)}-\sum_{i=0}^{k-1} \mathbf{u}_{i} \mathbf{u}_{i}^{*} \mathbf{w}^{(k)}}{\left\|\mathbf{w}^{(k)}-\sum_{i=0}^{k-1} \mathbf{u}_{i} \mathbf{u}_{i}^{*} \mathbf{w}^{(k)}\right\|}, \text { for all } 1 \leq k \leq d,
\end{aligned}
$$

where $d=C_{d} \log n$ with constant $C_{d}=\frac{1}{2 \log \left(\frac{{ } f+3}{4}\right)}+1$. $C_{f}$ will be defined later in Lemma II.4, and it does not depend on $n$ or $m$.

Since $d<m,\left\{\mathbf{u}_{i}\right\}_{i=0}^{d}$ is a set of $d+1$ orthogonal vectors in $\mathcal{C}^{m}$. By definition, $\mathbf{w}^{(k)} \in \operatorname{Sp}\left(\mathbf{u}_{0}, \mathbf{u}_{1}, \cdots, \mathbf{u}_{k}\right)$ for any $1 \leq k \leq d$ and $\mathbf{w}^{(k)}$ can be written as $\mathbf{u}_{i}$ :

$$
\mathbf{w}^{(k)}=\sum_{i=0}^{k} c_{i}^{(k)} \mathbf{u}_{i} .
$$


By writing $P_{L}\left[\mathbf{w}^{(k)} \odot \mathbf{y}\right]$ in the basis of $\mathbf{u}_{0}, \cdots, \mathbf{u}_{k+1}$ as $P_{L}\left[\mathbf{w}^{(k)} \odot \mathbf{y}\right]=\sum_{i=0}^{(k+1)} \tilde{c}_{k+1}^{(i)} \mathbf{u}_{i}$, the update formula (4) can be then rewritten as the update of $\left\{c_{i}^{(k)}\right\}_{i=0}^{k}$ as follows:

$$
\begin{aligned}
& \tilde{c}_{i}^{(k+1)}=\mathbf{u}_{i}^{*}\left[\left(\sum_{i=0}^{k} c_{i}^{(k)} \mathbf{u}_{i}\right) \odot \mathbf{u}_{0}\right], 0 \leq i \leq k \\
& \tilde{c}_{k+1}^{(k+1)}=\left\|P_{L}\left[\left(\sum_{i=0}^{k} c_{i}^{(k)} \mathbf{u}_{i}\right) \odot \mathbf{u}_{0}-\sum_{i=0}^{k} \tilde{c}_{i}^{(k+1)} \mathbf{u}_{i}\right]\right\| \\
& c_{i}^{(k+1)}=\frac{\tilde{c}_{i}^{(k+1)}}{\sqrt{\sum_{i=0}^{k+1} \tilde{c}_{i}^{(k+1) 2}}}, 0 \leq i \leq k+1 .
\end{aligned}
$$

While (6) seems complicated, this explicit formula will not be used later in the proof. Instead, the estimations

$$
0 \leq \tilde{c}_{k+1}^{(k+1)} \leq \sqrt{1-\sum_{i=0}^{k}\left|\tilde{c}_{i}^{(k+1)}\right|^{2}}
$$

and (10) (will be presented later) are sufficient, where the second inequality of (8) follows from the fact that $\sum_{i=0}^{k+1}\left|\tilde{c}_{i}^{(k+1)}\right|^{2}=\left\|P_{L}\left[\mathbf{w}^{(k)} \odot \mathbf{y}\right]\right\|^{2} \leq\left\|\mathbf{w}^{(k)} \odot \mathbf{y}\right\|^{2}=\|\mathbf{y}\|^{2}=1$.

The outline of the proof is as follows: first, we show that $\mathbf{u}_{i}$ can be well approximately by random vectors $\mathbf{v}_{i}$ from $C N(0, \mathbf{I} / m)$ in Lemma II.1. This step decouples the dependency between the sensing vectors and the estimations at the $k$-th iteration. Second, we investigate that the approximate dynamic of $\left\{c_{k}^{(i)}\right\}_{i=0}^{k}$ defined in (5) - (7), by replacing $\mathbf{u}_{i}$ with $\mathbf{v}_{i}$ in Lemma II.3 and II.4. Third, we obtain the dynamic of $\left\{c_{k}^{(i)}\right\}_{i=0}^{k}$ from applying a perturbation result in Lemma II.2 to the dynamic we obtained in the second step. Finally, we prove that at the $d$-th iteration, the estimation is already sufficiently good, and Lemma II.5, a variant of [27, Theorem 2], will be used to prove that the algorithm succeeds.

Lemma II.1. There exists $\left\{\mathbf{v}_{i}\right\}_{i=0}^{d}$ such that $\mathbf{v}_{i}$ are i.i.d. sampled from $C N(0, \mathbf{I} / m), \mathbf{u}_{0}=\mathbf{v}_{0} /\left\|\mathbf{v}_{0}\right\|$, and

$$
\begin{aligned}
& \operatorname{Pr}\left(\left\|\mathbf{u}_{k}-\mathbf{v}_{k}\right\|>\frac{\log m}{\sqrt{m}}\right)<C \exp \left(-C \log ^{2} m\right) \text { for } k=0,1 \\
& \operatorname{Pr}\left(\left\|\mathbf{u}_{k}-\mathbf{v}_{k}\right\|>2 \sqrt{\frac{n}{m}}\right)<C \exp (-C n) \text { for } 2 \leq k \leq d \\
& \operatorname{Pr}\left(\left|\tilde{c}_{k}^{(k)}\right|>2 \sqrt{\frac{n}{m}}\right)<C \exp (-C n) \text { for } 1 \leq k \leq d .
\end{aligned}
$$

In addition, we have the following properties:

$$
\left\|\mathbf{v}_{i}\right\| \leq 2,\left\|\mathbf{v}_{i}\right\|_{\infty} \leq \frac{\log m}{\sqrt{m}}, \text { for all } 0 \leq i \leq d
$$

holds with probability $1-2 m(d+1) \exp \left(-\log ^{2} m\right)-2 m(d+1) \exp \left(-\log ^{2} m / 4\right)$.

Lemma II.2. For any $\mathrm{x} \in \mathcal{C}^{m}$ and $\mathbf{x} \sim C N\left(0, \mathbf{I}_{m \times m} / m\right)$, with probability at least $1-m \exp (-n / 6)$, we have

$$
\frac{1}{m}\|\operatorname{phase}(\mathbf{x}+\mathbf{y})-\operatorname{phase}(\mathbf{x})\|_{1} \leq C \log m \max \left(\|\mathbf{y}\|, \frac{n}{m}\right)
$$

Lemma II.3. For any $\mathbf{x} \in \mathcal{C}^{m}$ defined by $\mathbf{x}=\sum_{i=0}^{d} c_{i} \mathbf{v}_{i}$, where $\sum_{i=0}^{d} c_{i}^{2}=1$ and $\mathbf{v}_{i} \sim C N(0, \mathbf{I} / m)$ for all $0 \leq i \leq d$. Define $f, g: \mathbb{R} \rightarrow \mathbb{R}$ by

$$
f(c)=\frac{1}{c} \mathbb{E}_{x_{0}, x_{1} \sim C N(0,1)} \frac{c x_{0}+\sqrt{1-c^{2}} x_{1}}{\left|c x_{0}+\sqrt{1-c^{2}} x_{1}\right|}\left|x_{0}\right| x_{0}^{*}
$$


and

$$
g(c)=\frac{1}{\sqrt{1-c^{2}}} \mathbb{E}_{x_{0}, x_{1} \sim C N(0,1)} \frac{c x_{0}+\sqrt{1-c^{2}} x_{1}}{\left|c x_{0}+\sqrt{1-c^{2}} x_{1}\right|}\left|x_{0}\right| x_{1}^{*},
$$

then

$$
\operatorname{Pr}\left(\left|\mathbf{v}_{0}^{*}\left[\mathbf{x} \odot \mathbf{v}_{0}\right]-f\left(\left|c_{0}\right|\right) c_{0}\right|<\frac{\log ^{2} m}{\sqrt{m}}\right)>1-\exp \left(-C \log ^{4} m\right),
$$

and for any $1 \leq j \leq d$,

$$
\operatorname{Pr}\left(\left|\mathbf{v}_{j}^{*}\left[\mathbf{x} \odot \mathbf{v}_{0}\right]-g\left(\left|c_{0}\right|\right) c_{j}\right|<\sqrt{\frac{n}{m}}\right)>1-\exp (-C n) .
$$

Lemma II.4. Given any $0<C_{0}<1$, there exists $C_{f}>1, C_{g}>0$ depending on $C_{0}$ such that

$$
\min _{0<c<C_{0}} f(c)>C_{f}, \min _{0<c<C_{0}} g(c)>C_{g} \text {. }
$$

In addition, we have

$$
\frac{f(c)}{g(c)} \geq 1, \text { for all } 0<c<C_{0} .
$$

The following lemma is a result of [27, Theorem 2]:

Lemma II.5. There exists $0<C_{0}<1, C_{1}^{\prime}, C_{2}^{\prime}>0$ such that if $\left|c_{0}^{\left(k_{0}\right)}\right|>C_{0}$ for some $k_{0}>0$, then the algorithm (4) converges to the solution with probability $1-\exp (-n / 2)-C_{1}^{\prime} \exp \left(-C_{2}^{\prime} m\right)$, in the sense that

$$
\lim _{k \rightarrow \infty} \inf _{\psi \in \mathbb{R}}\left\|e^{i \psi} \mathbf{z}-\mathbf{x}^{(k)}\right\|=0 .
$$

Lemma II.6. With probability at least $1-1 / \log n-\exp (-C n),\left|c_{0}^{(1)}\right| \leq \frac{1}{2 \sqrt{n \log n}}$.

For the rest of the proof, we first assume that for all $1 \leq k \leq d,\left|c_{0}^{(k)}\right|<C_{0}$, since otherwise Lemma II.5 already implies Theorem I.1. The goal is to show that under this assumption, we will have $\left|c_{0}^{(d+1)}\right|>C_{0}$, and then Lemma II.5 implies Theorem II.1.

Let $\mathbf{c}=\left\{c_{i}\right\}_{i=0}^{d} \in \mathcal{C}^{d+1}$, we choose a set of covering balls of radius $n / m$ in the set $\mathcal{S}=\left\{\mathbf{c} \in \mathcal{C}^{d+1}\right.$ : $\left.\|\mathbf{c}\|=1,\left|c_{0}\right| \leq C_{0}\right\}$. That is, we find a subset $\mathcal{S}_{0} \subset \mathcal{S}$ such that for any $\mathbf{c} \in \mathcal{S}$, there exists an element $\overline{\mathbf{c}}=\left\{\bar{c}_{i}\right\}_{i=0}^{d} \in \mathcal{S}_{0}$ such that $\|\mathbf{c}-\overline{\mathbf{c}}\| \leq n / m$. Following [26, Lemma 5.2], $\mathcal{S}_{0}$ can be chosen such that 
$\left|\mathcal{S}_{0}\right| \leq\left(1+\frac{2 m}{n}\right)^{2(d+1)}$. We assume that for all $\overline{\mathbf{c}} \in \mathcal{S}_{0}$, the property in Lemma II.2 holds for $\mathbf{x}=\sum_{i=0}^{d} \bar{c}_{i} \mathbf{v}_{i}$, and the property in Lemma $\Pi$ I.3 also holds. Then for all $j=0,1, \cdots, d$, we have

$$
\begin{aligned}
& \left|\mathbf{u}_{j}^{*}\left[\sum_{i=0}^{d} c_{i} \mathbf{u}_{i} \odot \mathbf{u}_{0}\right]-\mathbf{v}_{j}^{*}\left[\sum_{i=0}^{d} \bar{c}_{i} \mathbf{v}_{i} \odot \mathbf{v}_{0}\right]\right| \\
= & \left|\frac{1}{\left\|\mathbf{v}_{0}\right\|} \mathbf{u}_{j}^{*}\left[\sum_{i=0}^{d} c_{i} \mathbf{u}_{i} \odot \mathbf{v}_{0}\right]-\mathbf{v}_{j}^{*}\left[\sum_{i=0}^{d} \bar{c}_{i} \mathbf{v}_{i} \odot \mathbf{v}_{0}\right]\right| \\
= & \mid\left(\frac{1}{\left\|\mathbf{v}_{0}\right\|} \mathbf{u}_{j}^{*}-\mathbf{v}_{j}^{*}\right)\left[\sum_{i=0}^{d} c_{i} \mathbf{u}_{i} \odot \mathbf{v}_{0}\right] \\
& -\mathbf{v}_{j}^{*}\left[\left[\sum_{i=0}^{d} c_{i} \mathbf{u}_{i} \odot \mathbf{v}_{0}\right]-\left[\sum_{i=0}^{d} \bar{c}_{i} \mathbf{v}_{i} \odot \mathbf{v}_{0}\right]\right] \mid \\
\leq & \left\|\frac{1}{\left\|\mathbf{v}_{0}\right\|} \mathbf{u}_{j}^{*}-\mathbf{v}_{j}^{*}\right\|\left\|\mathbf{v}_{0}\right\| \\
& \left.+\left\|\mathbf{v}_{j}\right\|\left\|_{\infty}\right\| \operatorname{sign}^{d} \sum_{i=0}^{d} c_{i} \mathbf{u}_{i}\right)-\operatorname{sign}\left(\sum_{i=0}^{d} \bar{c}_{i} \mathbf{v}_{i}\right)\|\|_{1}\left\|\mathbf{v}_{0}\right\|_{\infty} \\
\leq & \left\|\mathbf{u}_{j}-\mathbf{v}_{j}\right\|+\left|\left\|\mathbf{v}_{0}\right\|-1\right|\left\|\mathbf{v}_{j}\right\| \\
& +\left\|\mathbf{v}_{j}\right\| \mathbf{v}_{\infty}\left\|\mathbf{v}_{0}\right\|{ }_{\infty} m \max \left(\sum_{i=0}^{d} c_{i}\left\|\mathbf{u}_{i}-\mathbf{v}_{i}\right\|+\left|c_{i}-\bar{c}_{i}\right|\left\|\mathbf{v}_{i}\right\|, \frac{n}{m}\right) \\
\leq & 2 \frac{\log m}{\sqrt{m}}+\log { }^{2} m\left(2 d \frac{n}{m}+d \max _{2 \leq i \leq d} 2\left|c_{i}\right| \sqrt{\frac{n}{m}}\right)+\left\|\mathbf{u}_{j}-\mathbf{v}_{j}\right\| .
\end{aligned}
$$

By the definition of $\mathcal{S}_{0}$, we have that for each $1 \leq k \leq d$, there exists $\overline{\mathbf{c}}^{(k)}=\left[\bar{c}_{0}^{(k)}, \cdots, \bar{c}_{d}^{(k)}\right] \in \mathcal{S}_{0}$ such that

$$
\sum_{i=0}^{k}\left|\bar{c}_{i}^{(k)}-c_{i}^{(k)}\right|^{2} \leq\left(\frac{n}{m}\right)^{2},\left|\bar{c}_{0}^{(k)}\right|<C_{0} .
$$

Combining the analysis in (15) (with c, $\overline{\mathbf{c}}$ replaced by $\mathbf{c}^{(k)}, \overline{\mathbf{c}}^{(k)}$ ), and applying (5) and Lemma II.4, we have that for $1 \leq j \leq k$,

$$
\begin{aligned}
& \left|\tilde{c}_{j}^{(k+1)}-g\left(\left|\bar{c}_{0}^{(k)}\right|\right) \bar{c}_{j}^{(k)}\right| \leq 4 \sqrt{\frac{n}{m}} \\
& +\log ^{2} m\left(2 d \max _{2 \leq i \leq k}\left|c_{i}^{(k)}\right| \sqrt{\frac{n}{m}}+2 d \frac{n}{m}\right)
\end{aligned}
$$

and for $j=0$,

$$
\begin{aligned}
& \left|\tilde{c}_{0}^{(k+1)}-f\left(\left|\bar{c}_{0}^{(k)}\right|\right) \bar{c}_{0}^{(k)}\right| \leq 3 \frac{\log m}{\sqrt{m}} \\
& +\log ^{2} m\left(2 d \max _{2 \leq i \leq k}\left|c_{i}^{(k)}\right| \sqrt{\frac{n}{m}}+2 d \frac{n}{m}\right) .
\end{aligned}
$$


Combining (16) and (17) with (8),

$$
\begin{aligned}
& \sqrt{\sum_{i=0}^{k+1}\left|\tilde{c}_{i}^{(k+1)}\right|^{2}} \geq \sqrt{\sum_{i=0}^{k}\left|\tilde{c}_{i}^{(k+1)}\right|^{2}} \\
\geq & \sqrt{f^{2}\left(\left|\bar{c}_{0}^{(k)}\right|\right)\left|\bar{c}_{0}^{(k)}\right|^{2}+g^{2}\left(\left|\bar{c}_{0}^{(k)}\right|\right)\left(1-\left|\bar{c}_{0}^{(k)}\right|^{2}\right)}-4(k+1) \sqrt{\frac{n}{m}} \\
& -2 d(k+1) \log ^{2} m\left(\max _{2 \leq i \leq k}\left|c_{i}^{(k)}\right| \sqrt{\frac{n}{m}}+\frac{n}{m}\right) \\
\geq & g\left(\left|\bar{c}_{0}^{(k)}\right|\right)-4(k+1) \sqrt{\frac{n}{m}} \\
& -2 d(k+1) \log ^{2} m\left(\max _{2 \leq i \leq k}\left|c_{i}^{(k)}\right| \sqrt{\frac{n}{m}}+\frac{n}{m}\right) .
\end{aligned}
$$

Combining (16) and (18) with the update formula (7), the estimation (11), and (14), using induction we can verify that for sufficiently large $n, m$, we have

$$
\max _{2 \leq j \leq k+1}\left|c_{j}^{(k+1)}\right|<\frac{4}{C_{g}} \sqrt{\frac{n}{m}}
$$

for all $0 \leq k \leq d-1$. By the assumption $m / \log ^{3} m \geq M n^{3 / 2} \log ^{1 / 2} n$ and Lemma II.6, we have

$$
\left|c_{0}^{(0)}\right|>\frac{C n \log ^{3} m}{m}
$$

Combining it with (17) and (8), it can be verified by induction that when $M$ is sufficiently large,

$$
\begin{aligned}
& \left|c_{0}^{(k+1)}\right| \geq\left|\tilde{c}_{0}^{(k+1)}\right| \geq \frac{C_{f}+1}{2}\left|\bar{c}_{0}^{(k)}\right| \geq \frac{C_{f}+1}{2}\left(\left|c_{0}^{(k)}\right|-\frac{n}{m}\right) \\
\geq & \frac{C_{f}+3}{4}\left|c_{0}^{(k)}\right| .
\end{aligned}
$$

for all $1 \leq 1 \leq k$.

As a result, combining it with Lemma II.6 we have

$$
\left|c_{0}^{(d+1)}\right| \geq\left(\frac{C_{f}+3}{4}\right)^{C_{d} \log n} \frac{1}{2 \sqrt{n \log n}}>C_{0}
$$

In fact, the second inequality requires

$$
C_{d}>\frac{\log C_{0}+\frac{1}{2} \log n+\log \log n}{\log \left(\frac{C_{f}+3}{4}\right) \log n},
$$

and for large $n$, our choice of $C_{d}=\frac{1}{2 \log \left(\frac{C_{f}+3}{4}\right)}+1$ would suffice. With (21), Lemma II.5 proves Theorem I.1.

In the end, we summarize the probability that the above analysis holds: the proof requires the events in all lemmas, and in addition, the events in Lemma II.2 and Lemma II.3 should hold for all $\mathbf{x}=\sum_{i=0}^{d} \bar{c}_{i} \mathbf{v}_{i}$, where $\overline{\mathbf{c}}=\left[\bar{c}_{0}, \bar{c}_{1}, \cdots, \bar{c}_{d}\right]$ is an element in $\mathcal{S}_{0}$. As a result, the probability is at least

$$
\begin{aligned}
& 1-2 C m d \exp \left(-\log ^{2} m\right)-\exp (-n / 2)-C_{1}^{\prime} \exp \left(-C_{2}^{\prime} m\right) \\
& -\log n-\exp (C n)-\left(1+\frac{2 m}{n}\right)^{2 d+2}(m \exp (-n / 6) \\
& \left.-\exp \left(-C \log ^{4} m\right)-d \exp (-C n)\right)
\end{aligned}
$$

which can be verified to converge to 1 as $n, m \rightarrow \infty$. 


\section{Proof of the Main Lemmas}

Proof of Lemma II.1. Since $L$ is a random $n$-dimensional subspace in $\mathcal{C}^{m}$, and $\Pi_{L}$ is a random projection matrix to $L, \mathbf{u}_{0}$ is random unit vector in $\mathcal{C}^{m}$ that is uniformly sampled from the sphere in $\mathcal{C}^{m}$. Therefore, it can be obtained through $\mathbf{v}_{0} \sim C N(0, \mathbf{I} / m)$ by

$$
\mathbf{u}_{0}=\frac{\mathbf{v}_{0}}{\left\|\mathbf{v}_{0}\right\|}
$$

Applying Lemma II.17 (with a scaling of $\sqrt{m}$ ) and $\left\|\mathbf{u}_{0}-\mathbf{v}_{0}\right\|=\left|\left\|\mathbf{v}_{0}\right\|-1\right|$, we proved (9) for $k=0$.

Under the $\sigma$-algebra generated by $\mathbf{u}_{0}$, the conditional distribution of $L$ is a random subspace generated by

$$
\operatorname{Sp}\left(\mathbf{u}_{0}\right) \oplus L_{0}
$$

where $L_{0}$ is a random $n$-1-dimensional subspace in the $m$-1-dimensional hyperplane $\operatorname{Sp}\left(\mathbf{u}_{0}\right)^{\perp}($ here $\oplus$ represents the direct sum of two subspaces). Since $\mathbf{w}^{(1)}$ is a random initialization on $L$ and $\mathbf{u}^{(1)}$ is the projection of $\mathbf{w}^{(1)}$ onto $\operatorname{Sp}\left(\mathbf{u}_{0}\right)^{\perp}, \mathbf{u}_{1}$ is a random unit vector on $L_{0}$. Combining it with the conditional distribution of $L_{0}, \mathbf{u}_{1}$ is a random unit vector that is orthogonal to $\mathbf{u}_{0}$. As a result, it can be generated from $\mathbf{v}_{1} \sim C N(0, \mathbf{I} / m)$ as follows:

$$
\mathbf{u}_{1}=\frac{\mathbf{v}_{1}-\mathbf{u}_{0} \mathbf{u}_{0}^{*} \mathbf{v}_{1}}{\left\|\mathbf{v}_{1}-\mathbf{u}_{0} \mathbf{u}_{0}^{*} \mathbf{v}_{1}\right\|}
$$

Since

$$
\operatorname{Pr}\left(\left|1-\left\|\mathbf{v}_{1}\right\|\right|>\frac{\log m}{3 \sqrt{m}}\right)<\exp \left(-C \log ^{2} m\right)
$$

and $\mathbf{u}_{0}^{*} \mathbf{v}_{1} \sim C N(0,1 / m)$ and as a result, Lemma $I$ I.18 with $m=1$ implies that

$$
\operatorname{Pr}\left(\left|\mathbf{u}_{0}^{*} \mathbf{v}_{1}\right|>\frac{\log m}{3 \sqrt{m}}\right)<\exp \left(-C \log ^{2} m\right) .
$$

Applying Lemma II.13, under the event of (23),

$$
\left\|\mathbf{u}_{1}-\frac{\mathbf{v}_{1}}{\left\|\mathbf{v}_{1}\right\|}\right\| \leq 2\left\|\mathbf{u}_{0} \mathbf{u}_{0}^{*} \mathbf{v}_{1}\right\| \leq \frac{2 \log m}{3 \sqrt{m}}
$$

combining it with (22), (9) with $k=1$ holds.

To prove (10), we first investigate the conditional distribution of $L$ under the $\sigma$-algebra generated by the algorithm so far, that is, generated by $\left\{\mathbf{u}_{i}\right\}_{i=0}^{k-1}$ and $\left\{\mathbf{w}_{i}\right\}_{i=0}^{k-1}$. That is, what is the conditional distribution of $L$ when $\left\{\mathbf{u}_{i}\right\}_{i=0}^{k-1}$ and $\left\{\mathbf{w}_{i}\right\}_{i=0}^{k-1}$ are fixed? Under this $\sigma$-algebra, $L$ satisfies the following properties:

$$
\begin{array}{r}
\mathbf{u}_{i} \in L, \quad 0 \leq i \leq k-1 \\
{\left[\mathbf{w}^{(i)} \odot \mathbf{y}\right]-\mathbf{w}^{(i+1)} \mathbf{w}^{(i+1) *\left[\mathbf{w}^{(i)} \odot \mathbf{y}\right] \perp L, 1} \leq i \leq k-2 .}
\end{array}
$$

The second property above holds since $\mathbf{w}^{(i+1)}$ is the normalization projection of $\mathbf{w}^{(i)} \odot \mathbf{y}$ onto $L$, and as a result, $\mathbf{w}^{(i+1)} \mathbf{w}^{(i+1) *}\left[\mathbf{w}^{(i)} \odot \mathbf{y}\right]=P_{L}\left[\mathbf{w}^{(i)} \odot \mathbf{y}\right]$. Recall that $L$ is a random $n$-dimensional subspace in $\mathcal{C}^{m}$, with this $\sigma$-algebra, its conditional distribution then can be written as

$$
L=\operatorname{Sp}\left\{\mathbf{u}_{i}\right\}_{i=0}^{k-1} \oplus L_{k}
$$

where $L_{k}$ is a random $n-k$-dimensional subspace in the $m-2 k+2$-space $R_{k}$ that is orthogonal to $\mathbf{u}_{i}$, $0 \leq i \leq k-1$ and $\left[\mathbf{w}^{(i)} \odot \mathbf{y}\right]-\mathbf{w}^{(i+1)} \mathbf{w}^{(i+1) *}\left[\mathbf{w}^{(i)} \odot \mathbf{y}\right], 1 \leq i \leq k-2$.

Since $\mathbf{w}^{(k)}$ is the projection of $\mathbf{w}^{(k-1)} \odot \mathbf{y}$ onto the subspace $L$ and $\mathbf{u}_{k}$ is the unit vector of the projection of $\mathbf{w}^{(k)}$ to the subspace orthogonal to $\operatorname{Sp}\left\{\mathbf{u}_{i}\right\}_{i=0}^{k-1}$, in conclusion, $\mathbf{u}^{(k)}$ is the unit vector that corresponds to the projection of $P_{R_{k}}\left[\mathbf{w}^{(k-1)} \odot \mathbf{y}\right]$ onto $L_{k}$, a random $n-k$-dimensional subspace in $R_{k}$. Applying Lemma II.8 (with $m, n, \mathcal{C}^{m}$ replaced by $m-2 k+2, n-k, R_{k}$ ), $\mathbf{u}_{k}$ can be written as

$$
\mathbf{u}_{k}=\sqrt{1-a^{2}} \mathbf{v}_{k}^{\prime}+a \frac{P_{R_{k}}\left[\mathbf{w}^{(k-1)} \odot \mathbf{y}\right]}{\left\|P_{R_{k}}\left[\mathbf{w}^{(k-1)} \odot \mathbf{y}\right]\right\|},
$$


where $\mathbf{v}_{k}^{\prime}$ is a unit vector on $R_{k}^{\prime}$, the $m-2 k+1$-dimensional subspace inside $R_{k}$ and orthogonal to $P_{R_{k}}\left[\mathbf{w}^{(k-1)} \odot \mathbf{y}\right]$, and $a$ is the length of the projection of $P_{R_{k}}\left[\mathbf{w}^{(k-1)} \odot \mathbf{y}\right] /\left\|P_{R_{k}}\left[\mathbf{w}^{(k-1)} \odot \mathbf{y}\right]\right\|$ onto $L_{k}$.

Since $L_{k}$ is a random subspace in $R_{k}, \mathbf{v}_{k}^{\prime}$ is a random unit vector on $R_{k}^{\prime}$ and can be derived through $\mathbf{v}_{k} \sim C N(0, \mathbf{I} / m)$ by

$$
\mathbf{v}_{k}^{\prime}=\frac{P_{R_{k}^{\prime}} \mathbf{v}_{k}}{\left\|P_{R_{k}^{\prime}} \mathbf{v}_{k}\right\|}
$$

Again use the fact that

$$
\operatorname{Pr}\left(\left|1-\left\|\mathbf{v}_{k}\right\|\right|>\frac{1}{6} \sqrt{\frac{n}{m}}\right)<\exp (-C n)
$$

and Lemma II.18 implies

$$
\operatorname{Pr}\left(\left\|\mathbf{v}_{k}-P_{R_{k}^{\prime}} \mathbf{v}_{k}\right\|>\frac{1}{6} \sqrt{\frac{n}{m}}\right)<2(2 k-1) \exp (-C n / 4(2 k-1)),
$$

and Lemma II.9 implies

$$
\operatorname{Pr}\left(a^{2}>2 \cdot \frac{n-k}{m-2 k+2}\right)<4 \exp (-C(n-k)) .
$$

Combining all estimations above with (24) and Lemma II.13, (10) is proved as follows:

$$
\begin{aligned}
& \left\|\mathbf{u}_{k}-\mathbf{v}_{k}\right\| \leq a+\sqrt{1-a^{2}}\left\|\mathbf{v}_{k}^{\prime}-\mathbf{v}_{k}\right\| \\
\leq & a+\sqrt{1-a^{2}}\left(\left|\left\|\mathbf{v}_{k}\right\|-1\right|+\left\|\mathbf{v}_{k}^{\prime}-\frac{\mathbf{v}_{k}}{\left\|\mathbf{v}_{k}\right\|}\right\|\right) \\
\leq & a+\left(\left|\left\|\mathbf{v}_{k}\right\|-1\right|+2\left\|P_{R_{k}^{\prime}} \mathbf{v}_{k}-\mathbf{v}_{k}\right\| /\left\|\mathbf{v}_{k}\right\|\right) \\
\leq & \sqrt{2 \cdot \frac{n-k}{m-2 k+2}}+\left(\frac{1}{6} \sqrt{\frac{n}{m}}+2 \frac{\frac{1}{6} \sqrt{\frac{n}{m}}}{1-\frac{1}{6} \sqrt{\frac{n}{m}}}\right) \leq 2 \sqrt{\frac{n}{m}}
\end{aligned}
$$

Note $\tilde{c}_{k}^{(k)}$ is the length of projection of $P_{R_{k}}\left[\mathbf{w}^{(k-1)} \odot \mathbf{y}\right]$ onto $L_{k}$, and $\left\|P_{R_{k}}\left[\mathbf{w}^{(k-1)} \odot \mathbf{y}\right]\right\| \leq \|\left[\mathbf{w}^{(k-1)} \odot\right.$ $\mathbf{y}]\|=\| \mathbf{y} \|=1$, by the definition of $a$ we have $\tilde{c}_{k}^{(k)} \leq a$. Then (25) implies (11).

At last, (12) is obtained by applying Lemma II.18 (with union bound and $m=1$ for the $\|\cdot\|_{\infty}$ norm).

Proof of Lemma II.2 It is based on a combination of Lemma II.11, II.12, and II.13, In particular, $t=$ $\max (\|\mathbf{y}\|, n / m)$ in Lemma II.11 (remark: there is a scaling factor of $\sqrt{m}$ between $\mathbf{x}$ in Lemma II.2 and Lemma II.11].

Proof of Lemma II.3. The proof is based on two components: first, we have

$$
\mathbb{E} \mathbf{v}_{0}^{*}\left[\mathbf{x} \odot \mathbf{v}_{0}\right]=f\left(\left|c_{0}\right|\right) c_{0},
$$

and for any $1 \leq j \leq d$,

$$
\mathbb{E} \mathbf{v}_{j}^{*}\left[\mathbf{x} \odot \mathbf{v}_{0}\right]=g\left(\left|c_{0}\right|\right) c_{j} .
$$

In fact, (26) follows directly from the definition of $f$, and (27) follows from the definition of $g$ as follows: that if we write $\mathbf{x}=c_{0} \mathbf{v}_{0}+\sqrt{1-\left|c_{0}\right|^{2}} \mathbf{v}^{\prime}$ with $\mathbf{v}^{\prime}=\frac{1}{\sqrt{\sum_{i=1}^{d}\left|c_{i}\right|^{2}}} \sum_{i=1}^{d} c_{i} \mathbf{v}_{i}$, then $\mathbf{v}^{\prime} \sim C N(0, \mathbf{I} / m)$ and the definition of $g$ implies

$$
\mathbb{E} \mathbf{v}^{\prime *}\left[\mathbf{x} \odot \mathbf{v}_{0}\right]=\sqrt{1-\left|c_{0}\right|^{2}} g\left(\left|c_{0}\right|\right)
$$

Noting that the correlation between $\mathbf{v}_{j}$ and $\mathbf{v}^{\prime}$ is $\frac{c_{j}}{\sqrt{1-\left|c_{0}\right|^{2}}}, 27$ is proved. 
Since each element of $\mathbf{v}_{0}$ is sampled from $C N(0,1 / m)$, it can be verified that the real component and the imaginary component of each entry of $\mathbf{x} \odot \mathbf{v}_{0}$ is sub-gaussian, with sub-gaussian parameter bounded above by $C / \sqrt{m}$. Applying Lemma II.14, this Lemma is proved.

Proof of Lemma II.4 We first let $f_{0}(c)=c f(c)$ and $g_{0}(c)=\sqrt{1-c^{2}} g(c)$, and show the following arguments:

$f_{0}$ and $g_{0}$ are bounded for $0 \leq c \leq C_{0}$.

$f_{0}, f_{0}^{\prime}$, and $g_{0}$ are Lipschitz continuous for $0 \leq c \leq C_{0}$.

$f_{0}^{\prime}(0)>1$.

Now we verify (28)-(30). First, by the rotational invariance of the complex normal distribution, $f_{0}$ can be equivalently defined by

$$
f_{0}(c)=\mathbb{E}_{x_{0}, x_{1} \sim C N(0,1)} \frac{x_{0}}{\left|x_{0}\right|}\left|c x_{0}+\sqrt{1-c^{2}} x_{1}\right|\left(c x_{0}+\sqrt{1-c^{2}} x_{1}\right)^{*} .
$$

It is clear that $f_{0}(c)=\mathbb{E}_{x_{0}, x_{1} \sim C N(0,1)}<\mathbb{E}\left(\left|x_{0}\right|+\left|x_{1}\right|\right)^{2}$ and is therefore bounded.

For all $0 \leq c, c^{\prime} \leq 1$,

$$
\left|c x_{0}+\sqrt{1-c^{2}} x_{1}-\left(c^{\prime} x_{0}+\sqrt{1-c^{\prime 2}} x_{1}\right)\right| \leq\left|c-c^{\prime}\right|\left|x_{0}\right|+\frac{\left|c-c^{\prime}\right|}{1-C_{0}^{2}}\left|x_{1}\right|,
$$

so we have

$$
\left|f(c)-f\left(c^{\prime}\right)\right| \leq\left|c-c^{\prime}\right| \mathbb{E} 2\left(\left|x_{0}\right|+\frac{1}{1-C_{0}^{2}}\left|x_{1}\right|\right)\left(\left|x_{0}\right|+\left|x_{1}\right|\right)
$$

and as a result, $f$ is Lipschitz continuous for $0 \leq c \leq C_{0}$

$f^{\prime}$ can be written as

$$
\begin{aligned}
& f^{\prime}(c)=\mathbb{E} \frac{x_{0}}{\left|x_{0}\right|}\left(\left(\left(\sqrt{1-c^{2}}-\frac{c^{2}}{\sqrt{1-c^{2}}}\right) x_{0}^{*} x_{1}+c\left|x_{0}\right|^{2}-c\left|x_{1}\right|^{2}\right) \times\right. \\
& \left.\frac{\left(c x_{0}+\sqrt{1-c^{2}} x_{1}\right)^{*}}{\left|c x_{0}+\sqrt{1-c^{2}} x_{1}\right|}+\left|c x_{0}+\sqrt{1-c^{2}} x_{1}\right|\left(x_{0}-\frac{c}{\sqrt{1-c^{2}}} x_{1}\right)^{*}\right)
\end{aligned}
$$

By Lemma II.13,

$$
\begin{aligned}
& \quad\left|\frac{c x_{0}+\sqrt{1-c^{2}} x_{1}}{\left|c x_{0}+\sqrt{1-c^{2}} x_{1}\right|}-\frac{c^{\prime} x_{0}+\sqrt{1-c^{\prime 2}} x_{1}}{\left|c^{\prime} x_{0}+\sqrt{1-c^{\prime 2}} x_{1}\right|}\right| \\
& \leq 2 \frac{\left|\left(c-c^{\prime}\right) x_{0}+\left(\sqrt{1-c^{2}}-\sqrt{1-c^{\prime 2}}\right) x_{1}\right|}{\left|c x_{0}+\sqrt{1-c^{2}} x_{1}\right|} \\
& \leq 2\left|c-c^{\prime}\right| \frac{\left|x_{0}\right|+\frac{1}{\sqrt{1-C_{0}^{2}}}\left|x_{1}\right|}{\left|c x_{0}+\sqrt{1-c^{2}} x_{1}\right|} .
\end{aligned}
$$

Considering that $\mathbb{E}\left|x_{0}\right|^{k_{1}}\left|x_{1}\right|^{k_{2}} /\left|c x_{0}+\sqrt{1-c^{2}} x_{1}\right|$ exists for all $k_{1}, k_{2} \geq 0$ (note that $\operatorname{Pr}\left(\left|c x_{0}+\sqrt{1-c^{2}} x_{1}\right|<\right.$ $r)<r^{2}$ as shown in Lemma II.10), one can show that $f^{\prime}$ is Lipschitz continuous for $0 \leq c \leq C_{0}$.

Similarly, one can prove that $g$ is bounded for $0 \leq c \leq 1$ and Lipschitz continuous for $0 \leq c \leq C_{0}$. Third,

$$
f_{0}^{\prime}(0)=\mathbb{E} \frac{x_{0}}{\left|x_{0}\right|}\left(x_{0}^{*}\left|x_{1}\right|+\left|x_{1}\right| x_{0}^{*}\right)=2 \mathbb{E}\left|x_{0}\right|\left|x_{1}\right| \approx 1.56>1 .
$$

Next, we will prove Lemma II.4 based on (28)-(30). To prove that $\min _{0<c<C_{0}} g(c)>C_{g}$, we first note that $g(c)$ is Lipschitz continuous since $g_{0}(c)$ and $\frac{1}{\sqrt{1-c^{2}}}$ are Lipschitz continuous. Then this can be 


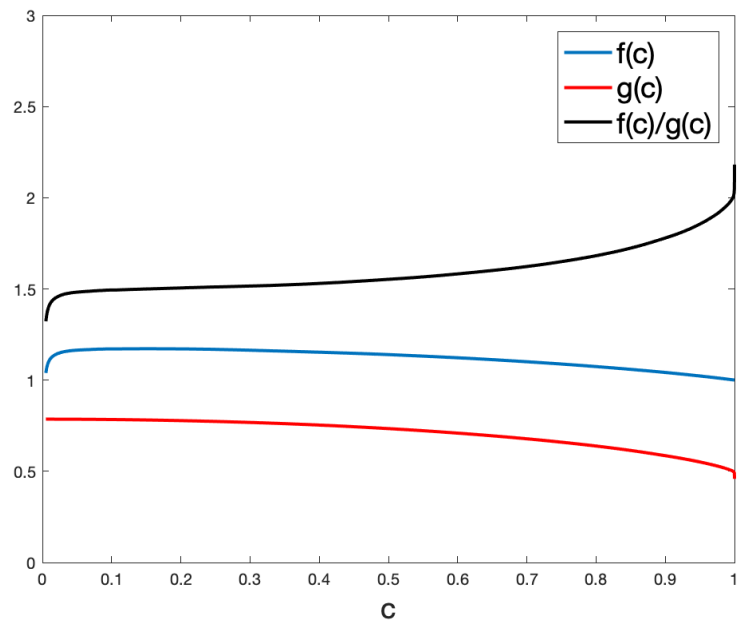

Fig. 1. The empirical values of $f, g$, and $f / g$.

verified numerically by calculating $g(c)$ for a sufficiently dense sampling of points in $0<c<C_{0}$. For example, if the Lipschitz constant of $g$ is $L_{g}$, and for $c=\left\{k C_{g} / L_{g}\right\}_{k=0}^{\left\lfloor\frac{L_{0} L_{g}}{C_{g}}\right\rfloor}, g(c)>2 C_{g}$, then we have $\min _{0<c<C_{0}} g(c)>C_{g}$. As shown in Figure II-C, such numerical verification is doable.

To prove $\min _{0<c<C_{0}} f(c)>C_{f}$ for some $C_{f}>1$, one first note that since $f(c)=\frac{f_{0}(c)-f_{0}(0)}{c-0}=$ $\int_{x=0}^{c} f_{0}^{\prime}(x), f_{0}^{\prime}(0)>1$ and the Lipschitz continuity of $f_{0}^{\prime}$ (assuming that the parameter is $L_{f_{0}^{\prime}}$ ), it shows that

$$
\min _{0<c<\frac{f_{0}^{\prime}(0)-1}{2 L_{f_{0}^{\prime}}}} f(c)>\frac{f_{0}^{\prime}(0)+1}{2} .
$$

It remains the show that $\min _{\frac{f_{0}^{\prime}(0)-1}{2 L_{f_{0}^{\prime}}}<c<C_{0}} f(c)>C_{f}$ for some $C_{f}>1$. The proof is then similar to the proof of $g$.

To prove (14), one may first use the same technique to verify that $\max _{0<c<C_{0}} g(c)<1$. Combining it with (13), 114) is proved.

We included the numerical values of $f(c), g(c)$, and $f(c) / g(c)$ in Figure II-C, to show that the inequalities (13) and (14) hold empirically, and as a result, the numerical verification method above would work.

Proof of Lemma II.5. For convenience, we first write down [27, Theorem 2] explicitly:

Theorem II.7 ([27], Theorem 2). There exists $C_{0}^{\prime}, C_{1}^{\prime}, C_{2}^{\prime}, M>0$ such that when $m>M n$, then with probability at least $1-C_{1}^{\prime} \exp \left(-C_{2}^{\prime} m\right)$, for any $\mathrm{x} \in \mathcal{C}^{n}$ such that

$$
\inf _{\psi \in \mathbb{R}}\left\|e^{i \psi} \mathbf{z}-\mathbf{x}\right\| \leq C_{0}^{\prime}\|\mathbf{z}\|
$$

then

$$
\inf _{\psi \in \mathbb{R}}\left\|e^{i \psi} \mathbf{z}-\mathbf{x}^{+}\right\| \leq \delta \inf _{\psi \in \mathbb{R}}\left\|e^{i \psi} \mathbf{z}-\mathbf{x}\right\|
$$

where $\mathrm{x}^{+}$is the vector obtained by applying one iteration of the standard alternating projection algorithm (without normalization) (1) to $\mathbf{x}$, and with $\left\{\mathbf{a}_{i}\right\}_{i=1}^{m}$ i.i.d. sampled from $C N(0, \mathbf{I})$.

By the analysis in Section II-A, (1) is equivalent to the algorithm that we are analyzing in (4) in terms of $\mathbf{w}^{(k)}$ as in $\mathcal{C}^{m}$. Therefore, [27, Theorem 2] implies that for $\mathbf{A}^{+}=\mathbf{A}^{*}\left(\mathbf{A}^{*} \mathbf{A}\right)^{-1}$, if

$$
\inf _{\psi \in \mathbb{R}}\left\|\mathbf{A}^{+}\left(e^{i \psi} \mathbf{u}_{0}-\mathbf{w}^{\left(k_{0}\right)}\right)\right\| \leq C_{0}^{\prime}\left\|\mathbf{A}^{+} \mathbf{u}_{0}\right\|
$$


then for any $k \geq k_{0}$,

$$
\inf _{\psi \in \mathbb{R}}\left\|\mathbf{A}^{+}\left(e^{i \psi} \mathbf{u}_{0}-\mathbf{w}^{(k+1)}\right)\right\| \leq \delta \inf _{\psi \in \mathbb{R}}\left\|\mathbf{A}^{+}\left(e^{i \psi} \mathbf{u}_{0}-\mathbf{w}^{(k)}\right)\right\| .
$$

Since $\mathbf{A}$ is a complex Gaussian, [11, Theorem 2.13] implies that the condition number of $\mathbf{A}$ is bounded with high probability:

$$
\operatorname{Pr}\left(\frac{\sigma_{\max }(\mathbf{A})}{\sigma_{\min }(\mathbf{A})} \leq \frac{\sqrt{m}+\sqrt{n}+t}{\sqrt{m}-\sqrt{n}-t}\right) \leq 1-2 \exp \left(-t^{2} / 2\right)
$$

Combining it (use $t=\sqrt{n}$ ) with $\inf _{\psi \in \mathbb{R}}\left\|e^{i \psi} \mathbf{u}_{0}-\mathbf{w}^{(k)}\right\|=\sqrt{\left(1-\left|c_{0}^{(k)}\right|\right)^{2}+\sum_{i=1}^{k}\left|c_{i}^{(k)}\right|^{2}}=\sqrt{\left(1-\left|c_{0}^{(k)}\right|\right)^{2}+1-\left|c_{0}^{(k)}\right|^{2}}$ there exists $0<C_{0}<1$ such that when $\left|c_{0}^{(k)}\right|>C_{0}$, $\inf _{\psi \in \mathbb{R}}\left\|\mathbf{A}^{+}\left(e^{i \psi} \mathbf{u}_{0}-\mathbf{w}^{(k)}\right)\right\|<C_{0}^{\prime}\left\|\mathbf{A}^{+} \mathbf{u}_{0}\right\|$ (note that $\mathbf{A}$ and $\mathbf{A}^{+}$have the same condition numbers), and then [27, Theorem 2] implies $\lim _{k \rightarrow \infty} \inf f_{\psi \in \mathbb{R}} \| \mathbf{A}^{+}\left(e^{i \psi} \mathbf{u}_{0}-\right.$ $\left.\mathbf{w}^{(k)}\right) \|=0$. Applying the fact that the condition number of $\mathbf{A}^{+}$is bounded again, we have $\lim _{k \rightarrow \infty} \inf _{\psi \in \mathbb{R}} \| e^{i \psi} \mathbf{u}_{0}-$ $\mathbf{w}^{(k)} \|=0$.

Proof of Lemma II.6. WLOG we may assume that $L=\operatorname{Sp}\left(\mathbf{e}_{1}, \cdots, \mathbf{e}_{n}\right)$ and $\mathbf{u}_{0}=\mathbf{e}_{1}$, and $\mathbf{w}^{(1)} \sim$ $C N\left(0, P_{L}\right)\left(\right.$ since $\mathbf{w}^{(1)}$ is a random vector on $\left.L\right)$. Then

$$
\left|c_{0}^{(1)}\right|=\frac{\left|w_{1}^{(1)}\right|}{\left\|\mathbf{w}^{(1)}\right\|} .
$$

Applying Lemma II.17 and note that $\left\|\mathbf{w}^{(1)}\right\|^{2}$ is the sum of $n$ unit complex gaussian squared, $\operatorname{Pr}\left(\left\|\mathbf{w}^{(1)}\right\|>\right.$ $2 \sqrt{n})<2 \exp (-C n)$; and Lemma II.10 implies that $\operatorname{Pr}\left(\left\|\mathbf{w}^{(1)}\right\|<1 / \sqrt{\log n}\right)<\log n$.

\section{Axillary Lemmas}

Lemma II.8. Assuming that projection of a unit vector $\mathrm{x} \in \mathcal{C}^{m}$ to a random n-dimensional subspace $L$ has length a, i.e., $\left\|P_{L} \mathbf{x}\right\|=a$, then

$$
\frac{P_{L} \mathbf{x}}{\left\|P_{L} \mathbf{x}\right\|}=a \mathbf{x}+\sqrt{1-a^{2}} \mathbf{v}
$$

where $\mathbf{v}$ is a unit vector perpendicular to $\mathbf{x}$, that is, $\mathbf{v}^{*} \mathbf{x}=1$.

Proof. Since $\frac{P_{L} \mathbf{x}}{\left\|P_{L} \mathbf{x}\right\|}$ is a unit vector, we may assume that

$$
\frac{P_{L} \mathbf{x}}{\left\|P_{L} \mathbf{x}\right\|}=b \mathbf{x}+\sqrt{1-b^{2}} \mathbf{v}
$$

where $\|\mathbf{v}\|=1$ and $\mathbf{v}^{*} \mathbf{x}=0$. It remains to prove $a=b$.

By the definition of projection, we have $\left(\mathbf{x}-P_{L} \mathbf{x}\right) \perp P_{L} \mathbf{x}$, i.e., $P_{L} \mathbf{x}^{*}\left(\mathbf{x}-P_{L} \mathbf{x}\right)=0$. Plug in the assumption (31) and $\left\|P_{L} \mathbf{x}\right\|=a$ we have

$$
\left(b \mathbf{x}+\sqrt{1-b^{2}} \mathbf{v}\right)^{*}\left((1-a b) \mathbf{x}-a \sqrt{1-b^{2}} \mathbf{v}\right)=0 .
$$

With $\mathbf{v}^{*} \mathbf{x}=0$ and $\|\mathbf{x}\|=\|\mathbf{v}\|=1$, it implies $b(1-a b)=a\left(1-b^{2}\right)$ and $a=b$.

Lemma II.9. Given a vector $\mathrm{x} \in \mathbb{R}^{m}$ and a random $n$-dimensional subspace $L$, then

$$
\begin{aligned}
& \operatorname{Pr}\left(\frac{1-\epsilon}{1+\epsilon} \leq \frac{m\left\|P_{L} \mathbf{x}\right\|^{2}}{n\|\mathbf{x}\|^{2}} \leq \frac{1+\epsilon}{1-\epsilon}\right) \geq \\
& 1-4 \exp \left(-c n \min \left(\frac{\epsilon^{2}}{C^{2}}, \frac{\epsilon}{C}\right)\right),
\end{aligned}
$$


Proof. WLOG we may assume that $\mathbf{x} \sim C N(0, \mathbf{I})$ and $L$ is the subspace spanned by the first $n$ standard basis $\mathbf{e}_{1}, \cdots, \mathbf{e}_{n}$. Then $\|\mathbf{x}\|^{2}=\sum_{i=1}^{m}\left|x_{i}\right|^{2}$ and $\left\|P_{L} \mathbf{x}\right\|^{2}=\sum_{i=1}^{n}\left|x_{i}\right|^{2}$. Applying Lemma II.17, we have

$$
\begin{aligned}
& \operatorname{Pr}\left((1-\epsilon) m \leq \sum_{i=1}^{m}\left|x_{i}\right|^{2} \leq(1+\epsilon) m\right) \\
\geq & 1-2 \exp \left(-c m \min \left(\frac{\epsilon^{2}}{C^{2}}, \frac{\epsilon}{C}\right)\right) \\
& \operatorname{Pr}\left((1-\epsilon) n \leq \sum_{i=1}^{n}\left|x_{i}\right|^{2} \leq(1+\epsilon) n\right) \\
\geq & 1-2 \exp \left(-c n \min \left(\frac{\epsilon^{2}}{C^{2}}, \frac{\epsilon}{C}\right)\right)
\end{aligned}
$$

Combining these two inequalities and $m \geq n$, the lemma is proved.

Lemma II.10. For $x \sim C N(0,1)$ and any $r>0, \operatorname{Pr}(|x| \leq r)<r^{2}$.

Proof. By the definition of $C N(0,1), \operatorname{Pr}(|x| \leq r)$ is the equivalent to $\operatorname{Pr}(\|\mathbf{y}\| \leq r)$ for $\mathbf{y} \in \mathbb{R}^{2}$ and sampled from $N\left(0, \mathbf{I}_{2 \times 2} / 2\right)$, which has a probability density function of $\frac{1}{\pi} \exp \left(-\|\mathbf{y}\|^{2}\right)$. This function is maximized at $\mathbf{y}=\mathbf{0}$ with a value of $1 / \pi$, and as a result, $\operatorname{Pr}(\|\mathbf{y}\| \leq r)<\pi r^{2} \cdot \frac{1}{\pi}=r^{2}$.

Lemma II.11. Given a vector $\mathbf{x} \in \mathbb{R}^{m}$ and $\mathbf{x} \sim C N\left(0, \mathbf{I}_{m \times m}\right)$. If $\mathbf{y} \in \mathbb{R}^{m}$ satisfies $\frac{1}{m} \sum_{i=1}^{m}\left|y_{i}\right|^{2} \leq t^{2}$ and $t \geq n / m$, then with probability at least $1-m \exp (-n / 6)$, we have

$$
\frac{1}{m} \sum_{i=1}^{m} \max \left(\frac{\left|y_{i}\right|}{\left|x_{i}\right|}, 1\right) \leq(4+\sqrt{2} l) t
$$

for $l=\left\lfloor-\log _{2} t\right\rfloor$.

Proof. WLOG we may rearrange the indices and assume that $\left|x_{1}\right| \leq\left|x_{2}\right| \leq \cdots \leq\left|x_{n}\right|$. Then Lemma II.12 implies that

$$
\operatorname{Pr}\left(\left|x_{j}\right|>\sqrt{j / 2 m}\right) \geq 1-\exp (-j / 6) .
$$

Applying a union bound,

$$
\operatorname{Pr}\left(\left|x_{j}\right|>\sqrt{j / 2 m} \text { for all } j \geq n\right) \geq 1-m \exp (-n / 6) .
$$

When the event in (33) holds, for all $j \geq n$ we have

$$
\sum_{i=j+1}^{2 j} \frac{\left|y_{i}\right|}{\left|x_{i}\right|} \leq \frac{1}{\left|x_{j}\right|} \sum_{i=j+1}^{2 j}\left|y_{i}\right| \leq \frac{1}{\left|x_{j}\right|} \sqrt{j \sum_{i=j+1}^{2 j}\left|y_{i}\right|^{2}} \leq m t \sqrt{2}
$$

Combining (34) for $j=t m, 2 t m, 4 t m, \cdots, 2^{l} t m$ ( $l$ is the largest integer such that $\left.2^{l} t<1\right)$ and $j=m / 2$, we have

$$
\sum_{i=t m+1}^{m} \frac{\left|y_{i}\right|}{\left|x_{i}\right|} \leq(2+l) m t \sqrt{2}
$$

In addition, it is clear that

$$
\sum_{i=1}^{t m} \max \left(\frac{\left|y_{i}\right|}{\left|x_{i}\right|}, 1\right) \leq t m
$$

Combining (35) and (36), the lemma is proved. (integer issue?) 


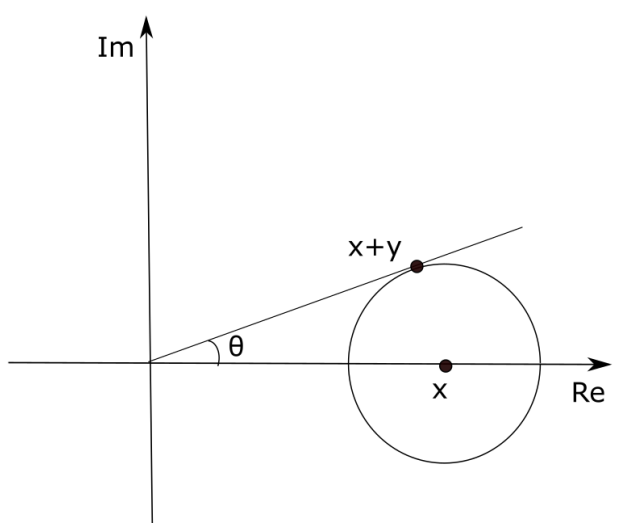

Fig. 2. Visualization of the proof of Lemma II.13 when $r<1$.

Lemma II.12. For a vector $\mathbf{x} \in \mathbf{C}^{m}$ and $\mathbf{x} \sim \sum C N\left(0, \mathbf{I}_{m \times m}\right)$, we have

$$
\operatorname{Pr}\left(\sum_{i=1}^{m} I\left(\left|x_{i}\right| \leq r\right)<2 r^{2} m\right)>1-\exp \left(-r^{2} m / 3\right) .
$$

Proof. We apply Lemma II.16 with $p=\operatorname{Pr}\left(\left|x_{1}\right| \leq r\right)$ and $\delta=2 r^{2} / p-1$. Applying Lemma II.10,

$$
\delta p=2 r^{2}-\operatorname{Pr}\left(\left|x_{1}\right| \leq r\right)>r^{2},
$$

which implies Lemma $\Pi .12$.

Lemma II.13. For any complex number $x, y \in \mathcal{C}$, $|\operatorname{phase}(x+y)-\operatorname{phase}(x)| \leq \min \left(2 \frac{|y|}{|x|}, 2\right)$. Similarly, for any vector $\mathbf{u}, \mathbf{v} \in \mathcal{C}^{m},\left\|\frac{\mathbf{u}+\mathbf{v}}{\|\mathbf{u}+\mathbf{v}\|}-\frac{\mathbf{v}}{\|\mathbf{v}\|}\right\| \leq \min \left(2 \frac{\|\mathbf{u}\|}{\|\mathbf{v}\|}, 2\right)$.

Proof. WLOG we only need to prove the first sentence and we may assume that $x=1$ and $|y|=r$. Then phase $(x)=e^{i 0}=1$, and on the complex plane, $x+y$ lies on a circle center at 1 with radius $r$.

When $r \geq 1$, $\mid$ phase $(x+y)-\operatorname{phase}(x) \mid$ is maximized when $y=-r$ and phase $(x+y)=-1$, then we have $|\operatorname{phase}(x+y)-\operatorname{phase}(x)|=2$.

When $r<1$, we would like to find a point on the circle such that its direction is as far from the direction of $\mathrm{x}$-axis as possible. As visualized in Figure II-D, $|\operatorname{phase}(x+y)-\operatorname{phase}(x)|$ is achieved when the line connecting $x+y$ and the origin is tangent to the circle. It implies that the maximal value is $\left|e^{i \theta}-1\right|$, where $\theta=\sin ^{-1} r$. Then we have the estimation $\left|e^{i \theta}-1\right|=2 \sin (\theta / 2)=\sin (\theta) / \cos (\theta / 2) \leq \sqrt{2} \sin (\theta)=\sqrt{2} r$ (the inequality uses the fact that $\theta \leq \pi / 2$ ).

Combining these two cases, Lemma II.13 is proved.

Lemma II.14 (Sum of sub-gaussian variables, Proposition 5.10 in [26]). Given $X_{1}, \cdots, X_{n}$ i.i.d. from a distribution with zero mean and sub-gaussian norm defined by $\|X\|_{\psi_{2}}=\sup _{p \geq 1} p^{-1 / 2}\left(\mathbb{E}|X|^{p}\right)^{1 / p}$, then

$$
\operatorname{Pr}\left(\left|\frac{1}{n} \sum_{i=1}^{n} X_{i}\right| \geq t\right) \leq \exp \left(-\frac{c n t^{2}}{\|X\|_{\psi_{2}}^{2}}+1\right)
$$

Lemma II.15 (Sum of sub-exponential variables, Corollary 5.17 in [26]). Given $X_{1}, \cdots, X_{n}$ i.i.d. from a distribution with zero mean and sub-exponential norm defined by $\|X\|_{\psi_{1}}=\sup _{p \geq 1} p^{-1}\left(\mathbb{E}|X|^{p}\right)^{1 / p}$, then

$$
\operatorname{Pr}\left(\left|\frac{1}{n} \sum_{i=1}^{n} X_{i}\right| \geq t\right) \leq 2 \exp \left(-c n \min \left(\frac{t^{2}}{\|X\|_{\psi_{1}}^{2}}, \frac{t}{\|X\|_{\psi_{1}}}\right)\right)
$$


Lemma II.16. $X_{1}, X_{2}, \cdots$ are i.i.d. Bernoulli variables with expectation $p$, then for any $\delta>1$,

$$
\operatorname{Pr}\left(\sum_{i=1}^{m} X_{i}>(1+\delta) p m\right) \leq \exp (-m \delta p / 3) .
$$

Proof. It follows from [20, Theorem 4,4] and the observation that when $\delta>1,(1+\delta) \log (1+\delta)>\frac{4}{3} \delta$.

Lemma II.17. For $\mathbf{v} \sim C N(0, \mathbf{I}), \operatorname{Pr}\left(\frac{1}{m}\left|\|\mathbf{v}\|^{2}-1\right|>t\right)<2 \exp \left(-c m \min \left(\frac{t^{2}}{C^{2}}, \frac{t}{C}\right)\right)$

Proof. We remark that $\|\mathbf{v}\|^{2}-m=\sum_{i=1}^{m}\left(\left(\Re\left(v_{i}\right)^{2}-1 / 2\right)+\left(\Im\left(v_{i}\right)^{2}-1 / 2\right)\right)$, and both $\Re\left(v_{i}\right)$ and $\Im\left(v_{i}\right)$ are i.i.d. sampled from $N\left(0, \frac{1}{2}\right)$. Since sub-gaussian squared is sub-exponential [26, Lemma 5.14] with mean $1 / 2 m$, and after centering, a sub-exponential distribution is still sub-exponential [26, Remark 5.18], $\Re\left(v_{i}\right)^{2}-\frac{1}{2}$ and $\Im\left(v_{i}\right)^{2}-\frac{1}{2}$ are i.i.d. sampled from a sub-exponential distribution with sub-exponential norm smaller than a constant $C$. Applying Lemma II.15, Lemma II.17] is proved.

Lemma II.18. For any $\mathbf{x} \sim C N\left(0, \mathbf{I}_{m \times m}\right), \operatorname{Pr}(\|\mathbf{x}\|>t) \leq 2 m \exp \left(-t^{2} / 4 m\right)$.

Proof. It follows from the classic tail bound: $\operatorname{Pr}(|N(0,1)|>t) \leq \exp \left(-t^{2} / 2\right)$, and a union bound of all real components of imaginary components of each element of $\mathbf{x}$, which are i.i.d. sampled from $N(0,1 / 2)$.

\section{DISCUSSIONS}

The current paper justifies the convergence of alternating minimization algorithm with random initialization for phase retrieval. Specifically, we demonstrate that it succeeds with $m / \log ^{3} m>M n^{1.5} \log ^{0.5} n$ for some $M>0$. A future direction is to find a better sample complexity, possibly via more sophisticated arguments: empirically, the algorithm succeeds with $m>O(n)$. It would also be interesting to compare the decoupling approach in this work and the leave-one-out approach in [10], both in phase retrieval and in broader settings.

\section{REFERENCES}

[1] S. Bahmani and J. Romberg. Phase Retrieval Meets Statistical Learning Theory: A Flexible Convex Relaxation. In A. Singh and J. Zhu, editors, Proceedings of the 20th International Conference on Artificial Intelligence and Statistics, volume 54 of Proceedings of Machine Learning Research, pages 252-260, Fort Lauderdale, FL, USA, 20-22 Apr 2017. PMLR.

[2] H. H. Bauschke, P. L. Combettes, and D. R. Luke. Hybrid projection-reflection method for phase retrieval. J. Opt. Soc. Am. A, 20(6):1025-1034, Jun 2003.

[3] M. Bayati and A. Montanari. The lasso risk for gaussian matrices. IEEE Transactions on Information Theory, 58(4):1997-2017, April 2012.

[4] T. T. Cai, X. Li, and Z. Ma. Optimal rates of convergence for noisy sparse phase retrieval via thresholded wirtinger flow. Ann. Statist., 44(5):2221-2251, 102016.

[5] E. J. Candès and X. Li. Solving quadratic equations via phaselift when there are about as many equations as unknowns. Foundations of Computational Mathematics, 14(5):1017-1026, 2014.

[6] E. J. Cands, X. Li, and M. Soltanolkotabi. Phase retrieval via wirtinger flow: Theory and algorithms. IEEE Transactions on Information Theory, 61(4):1985-2007, April 2015.

[7] E. J. Cands, T. Strohmer, and V. Voroninski. Phaselift: Exact and stable signal recovery from magnitude measurements via convex programming. Communications on Pure and Applied Mathematics, 66(8):1241-1274, 2013.

[8] A. Chai, M. Moscoso, and G. Papanicolaou. Array imaging using intensity-only measurements. Inverse Problems, $27(1): 015005,2011$.

[9] Y. Chen and E. Candes. Solving random quadratic systems of equations is nearly as easy as solving linear systems. In C. Cortes, N. D. Lawrence, D. D. Lee, M. Sugiyama, and R. Garnett, editors, Advances in Neural Information Processing Systems 28, pages $739-747$. Curran Associates, Inc., 2015.

[10] Y. Chen, Y. Chi, J. Fan, and C. Ma. Gradient Descent with Random Initialization: Fast Global Convergence for Nonconvex Phase Retrieval. mar 2018 .

[11] K. R. Davidson and S. J. Szarek. Local operator theory, random matrices and Banach spaces. In Handbook of the geometry of Banach spaces, Vol. I, pages 317-366. North-Holland, Amsterdam, 2001.

[12] J. R. Fienup. Reconstruction of an object from the modulus of its fourier transform. Opt. Lett., 3(1):27-29, Jul 1978.

[13] J. R. Fienup. Phase retrieval algorithms: a comparison. Appl. Opt., 21(15):2758-2769, Aug 1982.

[14] R. W. Gerchberg and W. O. Saxton. A practical algorithm for the determination of the phase from image and diffraction plane pictures. Optik (Jena), 35:237+, 1972.

[15] T. Goldstein and C. Studer. PhaseMax: Convex Phase Retrieval via Basis Pursuit. 2016. 
[16] D. Gross, F. Krahmer, and R. Kueng. A partial derandomization of phaselift using spherical designs. Journal of Fourier Analysis and Applications, 21(2):229-266, 2015.

[17] P. Hand and V. Voroninski. An Elementary Proof of Convex Phase Retrieval in the Natural Parameter Space via the Linear Program PhaseMax. 2016.

[18] P. Hand and V. Voroninski. Corruption Robust Phase Retrieval via Linear Programming. dec 2016.

[19] S. Marchesini, Y.-C. Tu, and H.-T. Wu. Alternating projection, ptychographic imaging and phase synchronization. Applied and Computational Harmonic Analysis, 41(3):815 - 851, 2016.

[20] M. Mitzenmacher and E. Upfal. Probability and computing: Randomized algorithms and probabilistic analysis. Cambridge university press, 2005.

[21] P. Netrapalli, P. Jain, and S. Sanghavi. Phase retrieval using alternating minimization. IEEE Transactions on Signal Processing, 63(18):4814-4826, Sept 2015.

[22] F. Salehi, E. Abbasi, and B. Hassibi. Learning without the phase: Regularized phasemax achieves optimal sample complexity. In S. Bengio, H. Wallach, H. Larochelle, K. Grauman, N. Cesa-Bianchi, and R. Garnett, editors, Advances in Neural Information Processing Systems 31, pages 8654-8665. Curran Associates, Inc., 2018.

[23] Y. Shechtman, Y. C. Eldar, O. Cohen, H. N. Chapman, J. Miao, and M. Segev. Phase retrieval with application to optical imaging: A contemporary overview. IEEE Signal Processing Magazine, 32(3):87-109, May 2015.

[24] M. Soltanolkotabi. Structured signal recovery from quadratic measurements: Breaking sample complexity barriers via nonconvex optimization. feb 2017.

[25] J. Sun, Q. Qu, and J. Wright. A geometric analysis of phase retrieval. In 2016 IEEE International Symposium on Information Theory (ISIT), pages 2379-2383, July 2016.

[26] R. Vershynin. Introduction to the non-asymptotic analysis of random matrices. Arxiv preprint arxiv:1011.3027, 2010.

[27] I. Waldspurger. Phase retrieval with random gaussian sensing vectors by alternating projections. IEEE Transactions on Information Theory, 64(5):3301-3312, May 2018.

[28] I. Waldspurger, A. d'Aspremont, and S. Mallat. Phase recovery, maxcut and complex semidefinite programming. Mathematical Programming, 149(1):47-81, 2015.

[29] G. Wang and G. Giannakis. Solving random systems of quadratic equations via truncated generalized gradient flow. In D. D. Lee, M. Sugiyama, U. V. Luxburg, I. Guyon, and R. Garnett, editors, Advances in Neural Information Processing Systems 29, pages 568-576. Curran Associates, Inc., 2016.

[30] H. Zhang, Y. Chi, and Y. Liang. Provable non-convex phase retrieval with outliers: Median truncated wirtinger flow. In Proceedings of the 33rd International Conference on International Conference on Machine Learning - Volume 48, ICML'16, pages 1022-1031. JMLR.org, 2016.

[31] H. Zhang and Y. Liang. Reshaped wirtinger flow for solving quadratic system of equations. In D. D. Lee, M. Sugiyama, U. V. Luxburg, I. Guyon, and R. Garnett, editors, Advances in Neural Information Processing Systems 29, pages 2622-2630. Curran Associates, Inc., 2016.

[32] T. Zhang. Phase retrieval using alternating minimization in a batch setting. jun 2017. 